\title{
Advances in Microecological Agents and Traditional Chinese Medicine in Reversing Cirrhosis
}

\author{
Juan $\mathrm{Liu}^{1, *}, \mathrm{Li} \mathrm{Liu}^{2}$ \\ ${ }^{1}$ Department of Medicine, Shaanxi University of Traditional Chinese Medicine (Xianyang 712000), China. \\ ${ }^{2}$ Department of Digestive Medicine, Affiliated Hospital of Shaanxi University of Traditional Chinese Medicine (Xianyang 712000 ), \\ China.
}

How to cite this paper: Juan Liu, Li Liu. (2020) Advances in Microecological Agents and Traditional Chinese Medicine in Reversing Cirrhosis. International Journal of Clinical and Experimental Medicine Research, 4(4), 272-277. DOI: 10.26855/ijcemr.2020.10.025

Received: October 28, 2020

Accepted: November 22, 2020

Published: December 2, 2020

*Corresponding author: Juan Liu, Department of Medicine, Shaanxi University of Traditional Chinese Medicine (Xianyang 712000), China.

Email: 1373150614@qq.com

\begin{abstract}
Liver cirrhosis (LC) is characterized by persistent, diffuse inflammation, fibrosis and intrahepatic structure. Remodeling as a characteristic manifestation of chronic liver disease. The routine treatment is mainly antiviral, but its treatment method is relatively limited, and the therapeutic effect is not ideal. in recent years, a large number of clinical data have shown that the use of microecological agents and traditional Chinese medicine treatment can inactivate hepatic stellate cells (HSC) and alleviate liver fibrosis, thus achieving the possibility of liver cirrhosis reversal. In this paper, the research progress on the reversibility of cirrhosis in recent years is reviewed.
\end{abstract}

\section{Keywords}

Cirrhosis, Reversal, Microecological Agents, Chinese Medicine, Antiviral, Treatment

\section{Introduction}

LC formation is a post-injury repair response that occurs in patients with chronic liver injury. Epidemiological statistics show that 350 million people worldwide are infected with hepatitis B virus, while nearly 25 per cent of hepatitis B patients in China develop into LC. HSC activation is the central link in LC formation, HSC plays a central role in the development of liver fibrosis after activation from the resting state. Collagen and other extracellular matrix (ECM) components in activated HSC are deposited on the liver parenchyma and vascular structure and damage them. Therefore, HSC inactivation can lead to decreased fibrosis activity and is a potential entry point [1] for anti-fibrotic therapy. HSC activation pathway can be interrupted and ECM degraded in the early stage of cirrhosis formation, which is a good time to reverse cirrhosis. The ECM in the later stage of liver cirrhosis is not stone, it may still be swallowed and degraded by macrophages in vivo, but the process of natural degradation of ECM is very slow. A large number of clinical data show that the use of integrated Chinese and western medicine therapy, dialectical treatment, moderate conditioning, can effectively improve the tissue structure of liver cirrhosis, slow down the process of liver cirrhosis, promote ECM degradation, and even transform theolobules were transformed into the structure and function of normal hepatic lobules. In this paper, we discuss the status and prospect of microecological agents and the role of traditional Chinese medicine in the reversal of cirrhosis.

\section{Antiviral Treatment}

Antiviral therapy is the key to the treatment of liver cirrhosis. Development of liver fibrosis is a dynamic process. As long as viral replication is effectively controlled and the HSC is at rest, liver fibrosis can reach the possibility of 
reversal, which is closely related to the level of liver fibrosis, the lower the degree of lesion, the greater the possibility of reversal. Hepatitis B (HBV) cirrhosis antiviral therapy: interferon treatment [2] at present, China's interference is known as: IFN-a, its course of treatment for one year, if after 12 weeks of treatment HBsA g quantitative decline after discontinuation IFN-a, use nucleoside (acid) analogue (NAs) treatment [3]. Communicate fully with patients before treatment on the advantages of IFN use and related adverse drug reactions, as well as the possibility of liver cirrhosis progression. When NAs are selected for treatment, entecavir (ETV) is recommended as the preferred drug. To consider the possible risk of withdrawal, the NAs treatment of patients with hepatitis B cirrhosis should be carried out as long as possible without considering the [4] of withdrawal, and it should be polyethylene glycol interferon should be made clear. Antiviral therapy for hepatitis c (HCV) cirrhosis: pegylated interferon combined with ribavirin (PR); direct antiviral drugs against HCV (DAAs); initial treatment of HCV gene type 1: sophibovir/redipavir. On the basis of anti-HIV, patients should combine lifestyle intervention, combined with polyene phospholipids, reduced glutathione, potassium magnesium aspartate, and use glycyrrhizic acid, adenylyl methionine and other drugs to protect liver, detoxify, anti-inflammatory, anti-enzyme, melatonin and so on during the active period of hepatitis inflammation. Although the above treatment is widely used in clinic, it is undeniable that the current use of various antiviral drugs its anti-fibrosis treatment related to the role and side effects challenges still exist, the therapeutic effect is not ideal. Microecological agents and traditional Chinese medicine have various ways of anti-fibrosis, and have achieved good results in many clinical applications, which is worthy of our further study and exploration.

\section{Treatment and Prevention of Cirrhosis from the Perspective of Microecological Agents}

The intestine is tightly connected to the liver through the portal circulation. Although the vast majority of intestinal bacteria are located in the intestinal cavity and the outer mucus layer, some intestinal microbes and their products may destroy the intestinal epithelial barrier and enter the liver quickly. Recent studies have found that gut microbiota is associated with liver disease. Gut microbiota-induced proinflammatory gene expression plays a central role in liver disease and provides an important target [5] for liver cirrhosis therapy. Therefore, the treatment or prevention of liver disease can delay the development of liver cirrhosis and even achieve the purpose of reversal. At present, the study of regulating intestinal flora mainly focuses on the [6] of probiotics. Probiotics use probiotics as potential therapy. Probiotics use reduces the risk of intestinal bacterial overgrowth, reduces inflammatory levels, and regulates liver inflammation by balancing pro-inflammatory cytokines and anti-inflammatory cytokines. HSC inactivation is restored to rest, ECM degradation, endotoxemia and bacterial translocation risk are reduced, and fibrosis is improved. Zhai Yuf eng et al. [7] used carbon tetrachloride (CCl4) method to establish a model of liver fibrosis in mice. The results showed that the level of serum transaminase decreased and the liver function improved. The mechanism may be that probiotics can effectively inhibit or reduce the activation of HSC expression, promote ECM degradation, and then inhibit or alleviate hepatocyte apoptosis, protect liver tissue and block the process of cirrhosis. Clinical studies have shown that patients with cirrhosis are treated with probiotics. Detection of liver function index found that blood ammonia level decreased, liver function improved, its mechanism may be to reduce the formation of endotoxin, reduce intestinal mucosal permeability, and then achieve the [8] of inhibiting the development of liver cirrhosis. At present, the commonly used probiotics mainly include probiotics, bifidobacterium, a variety of probiotics mixture (vsl\#3).

3.1 Probiotic lactulose is one of the preb the growth of intestinal beneficial bacteria. Studies have shown that lactulose can reduce intestinal permeability and inhibit endotoxemia in patients with cirrhosis. Mechanism is to promote the growth of bifidobacterium, inhibit the survival of harmful flora, and regulate the balance of intestinal microbes, thus reducing the permeability [9] of intestinal mucosa.

3.2 Bifidobacterium bifidobacterium tetralogy tablets contain Bifidobacterium, Lactobacillus and other physiological bacteria,The above components can form a natural intestinal biological barrier, repair damaged physical barriers, and reduce intestinal mucosal permeability, The inhibition of inflammatory response, the prevention of bacterial translocation and the restoration of healthy balance of host intestinal tract are of great significance [10] HSC inactivation and liver cirrhosis reversal.

$3.3 \mathrm{vsl} \# 3$ is a multi-species probiotic bacterium consisting of eight strains of bacteria (Streptococcus thermophilus, Bifidobacterium short, Bifidobacterium long, Bifidobacterium infant, Lactobacillus acidophilus, Lactobacillus plantarum, Lactobacillus paracetamol and Lactobacillus nigra).the combination of several bacteria and strains can synergistically produce more significant effects than a single strain. And in a nonalcoholic steatohepatitis (NASH) mouse model, VSL\#3 have been shown to reduce fibrosis and promote ECM degradation by interrupting HSC ac- 
tivation pathways in patients with various liver diseases, including cirrhosis, thus restoring normal liver tissue structure [11].

Microbial agents are becoming part of many treatments for liver diseases because their effects on intestinal flora can have a positive effect on many liver diseases. However, it will be difficult to validate this treatment from the perspective of evidence-based medicine, because different microecological agents can have different effects in different diseases. As the research of microbial agents is increasing, the prospect in this field is optimistic [12]. Gut microbiota can produce a diverse range of compounds that play a major role in regulating the activity of distal organs and the liver is strategically positioned downstream of the gut. We are gaining increased insight into the close relationship between the gut and the liver evoked by systemic metabolic changes. The evaluation of the gut-microbiota-liver metabolic network and the intervention of these relationships using probiotics, synbiotics, prebiotics with sufficient nutrition might aid the development of treatment and prevention for liver cirrhosis patients [13]. An accumulating body of research suggests that the disparate observations in liver disease-related studies could be unified and explained by the microbiome. It is now widely accepted that liver damage is a result of an extensive interplay between gut microbiota via specialized molecules such as TMA, acetaldehyde, LPS and host-immune system via Kupffer cells-mediated liver inflammation. However, a comprehensive understanding of the exchange between the microbiome and the liver still evades us. Animal models, particularly rodents, have been instrumental in elucidating many important mechanistic pathways in disease etiology. The introduction of the microbiome into these models will provide a more complete view of the cancer ecosystem. Because microbiome research is sensitive to technical variability that often masks underlying biological signal, there is a need for consistency in technical platforms and standardized protocols, so that findings from different laboratories (and model organisms) can be replicated and validated. Additionally, it is also critical to use an animal model that mimics human disease as closely as possible in all its physiological and metabolic manifestations [14].

\section{Treatment and Prevention of Cirrhosis from the Perspective of Chinese Medicine}

Chinese herbal medicine has been widely used in China since ancient times, and its medicinal value has been strongly supported by clinical application. In Chinese medicine, herbs may play a different role in cirrhosis, such as scavenging free radicals, inhibiting lipid peroxidation, increasing glutathione content, improving liver microcirculation. The mechanism [13] of interrupting HSC activation pathway, promoting ECM degradation and repairing damaged hepatocytes. The composition of Chinese herbal medicine is complicated, its action mechanism and active ingredient are difficult to fully elucidate. Nevertheless, it can exert anti-hepatic fibrosis effect through many kinds [14] cytokines and cell signaling pathway.TCM syndrome differentiation and treatment, emphasis on individualized treatment, through the traditional Chinese medicine prescription, single medicine, Chinese patent medicine in liver cirrhosis reversal treatment showed obvious advantages.

\subsection{The role of TCM prescription in the treatment of cirrhosis reversal}

Gentian Xiegan decoction has the effect of clearing the viscera and heat, clearing the liver and gallbladder and solid fire, and clearing the liver through dampness and heat. Prescription: Gentiana, Gardenia, Radix Scutellariae, Mutong, Alisma, Plantago, Bupleurum, Glycyrrhiza, Angelica sinensis, Radix Rehmanniae, Castanopsis, Oyster, Turtle Plate. Wang Dianbing and other [15] used Gentian Xiegan decoction and Biejia decoction to treat cirrhosis. The results showed that the total clinical efficiency of the inquiry group was statistically significant $(\mathrm{P}<0.05)$, the liver function of the patients was improved well, and the HSCs activation was inhibited. Professor Zhou Daqiao referred to a large number of ancient and modern literature and combined with his own many years of clinical experience to create soft liver decoction to treat patients with cirrhosis, its therapeutic effect is remarkable. Soft liver soup group became: vinegar Biejia, Panax notoginseng, peach kernel, salvia miltiorrhiza, astragalus, stir-fried Atractylodes macrocephala, Poria cocos, tulip, fried Fructus Aurantii, half lotus, Schisandra chinensis, leaf beads 12 kinds of drugs, the drugs match, dispelling evil to help the right, attack and complement each other, to achieve the soft liver Sanjie, promoting blood stasis, invigorating qi and nourishing yin. In the treatment of liver fibrosis, blocking the development of liver cirrhosis has achieved a definite effect. The mechanism may be that soft liver decoction inhibits the HSC activation pathway, eliminates the inducing factors of liver fibrosis and cirrhosis, blocks ECM synthesis and promotes its degradation, thus achieving the [16] of reversing liver fibrosis and preventing the formation of cirrhosis. Yinchenhao decoction has definite effect in treating hepatobiliary damp-heat syndrome of cholestatic liver disease. Its prescription is: Yinchen, Gardenia jasminoides, Artemisia annua, rhubarb. It has been found that Yinchen Artemisia decoction can improve liver fibrosis and liver function by regulating bile acid metabolism and related signaling pathways. The mechanism is that Yinchen Artemisia decoction regulates the synthesis, 
secretion and excretion of bile acids to make bile acid metabolism nearly normal, reduce its accumulation in the liver, and then alleviate and block liver fibrosis. The activation of HSC was blocked by transforming growth factor $\beta 1$ (TGF- $\beta 1$ )/Smad/EPK signaling pathway, which reduced the apoptosis and injury of hepatocytes and made the reversal of liver cirrhosis possible [17-18]. Some studies have pointed out that rhubarb promotes HSC apoptosis and plays an important role in reducing liver fibrosis [19-20].

\subsection{The role of monotherapy in reverse therapy of cirrhosis}

Schisandra acid, sweet, warm sex, to the lung, heart, kidney meridian. Hu Yanwu et al. [21] used CCl4 rat liver injury model control test. Detection of rat serum showed that liver function improved significantly, liver fibrosis decreased significantly, confirmed that Schisandra chinensis stem extract has a good role in protecting liver function and anti-hepatic fibrosis. The mechanism is to reduce the expression of TGF- $\beta 1$, hinder the activation of HSCs, reduce the expression of $\alpha$ - smooth muscle actin ( $\alpha$-SMA), promote ECM degradation, and then prevent liver fibrosis and achieve the possible [22] of liver cirrhosis reversal. Schisandra chinensis has antioxidant, anti-inflammatory, anti-tumor and liver protection effects. The effects of Schisandra chinensis on HSC activation were observed. It was found that Schisandra chinensis could significantly inhibit Smad phosphorylation stimulated by TGF- $\beta 1$ and signal transduction of mitogen activated protein kinase and reduce ECM deposition, so it prevented the progression of liver fibrosis. Hedyotis diffusa bitter, light, cold sex, has the effect of eliminating carbuncle and dispersing knot, clearing heat and detoxifying [23]. Studies have shown that [24] extract of Hedyotis diffusa can effectively reduce the degree of liver fibrosis and protect liver tissue. Salvia miltiorrhiza bitter, sex slightly cold, return heart, liver classics. Cao Haifang and other [25] selected 106 patients with hepatitis B cirrhosis as the object of study, the observation group on the basis of tenofovirate patients plus Salvia miltiorrhiza injection. The results of the control experiment showed that the injection of Salvia miltiorrhiza in patients with hepatitis B cirrhosis significantly improved the progression of liver fibrosis and changed the liver function and fibrosis in a virtuous circle. More studies have found that Salvia miltiorrhiza can eliminate oxygen free radicals in the body and tissue, and play an inhibitory role in hepatocyte degeneration and necrosis,And can promote the degradation ECM liver cells, so that the body fibrosis tissue resolution. On the other hand, Salvia miltiorrhiza improves cell secretion of interstitial collagenase. At the same time, it can inhibit the ECM production rate and accelerate the reabsorption of liver fibrosis in the body, and thus achieve the possible [26-27] of inhibiting the occurrence, development and reversal of liver fibrosis. Biejia taste salty, cold, to the liver and kidney meridian. Studies have shown that Biejia has a definite clinical effect on cirrhosis. Cirrhosis patients with symptoms, tongue, pulse, increase serum albumin, reduce the spleen, there is a certain improvement in prognosis, prevent the progression of liver cirrhosis effect [28].

\subsection{Role of Chinese patent medicines in the treatment of cirrhosis reversal}

Sun Zhenliang and other [29] established a CCL4 induced liver fibrosis model in rats. The results showed that the compound Biejia soft liver tablet had a significant protective effect on liver function rat liver fibrosis model, The possible mechanism is related to inhibiting the expression of COX-2, $\alpha$-SMA protein in liver tissue and hindering the activation of HSCs. According to some studies, the [31] of Fuzheng Huayu recipe was 90.7\%, 78.8\% and $60.0 \%$ respectively. There was significant difference in the number of cases of liver fibrosis decline and aggravation between Fuzheng Huayu prescription group and other drug use groups. The results showed that the LSM evaluation of anti-hepatic fibrosis was not affected by the changes of ALT, AST, GGT and PT, INR [32], such as li aizhi, on the basis of routine western medicine treatment, 25 patients with this disease were treated with consistent decoction (including salvia miltiorrhiza, medlar, raw Rehmannia glutinosa, Ophiopogon japonicus, azadirachta, Angelica sinensis, etc.) with a total effective rate of $96.0 \%$, suggesting that the drug can effectively inhibit the progression of liver disease and delay the progression of disease deterioration. Gu Shaoyu [33] treated 72 patients with early cirrhosis with self-made Qingfu soft liver decoction. The drug composition: Salvia miltiorrhiza, Lycium barbarum, Astragalus membranaceus, Potentilla chinensis, Artemisia annua, Codonopsis, Poria cocos, Atractylodes macrocephala, Biejia, Angelica sinensis, Trionyx sinensis, Trionyx sinensis, Radix Rehmanniae, Curcuma, Gardenia, Panax notoginseng powder. The results showed that the total effective rate was $93.06 \%$, indicating that the prescription had the effect of removing dampness and detoxifying, activating blood and removing blood stasis, tonifying deficiency and tonifying qi, and had better curative effect on the disease.

\section{Summary}

From the current medical development, the irreversible conclusion of cirrhosis is not accurate enough, but also overdue. A large number of clinical experience data show that a considerable number of patients HSC inactivated 
after treatment with microecological agents and traditional Chinese medicine, ECM degradation, matrix metalloproteinase expression was inhibited, esophageal varices were significantly improved, and even portal hypertension was reversed, thus achieving the most effective treatment, making the reversal of cirrhosis possible. The mechanism of anti-hepatic fibrosis of traditional Chinese medicine involves many links, levels and targets in the pathophysiological process of liver fibrosis. Compared with western medicine with single target, the comprehensive pharmacological action characteristics of traditional Chinese medicine show unique advantages. It is believed that Chinese medicine can bring innovation and hope for breakthrough in liver fibrosis treatment. Chemistry, pharmacology, molecular biology and other multi-disciplinary technology in-depth study of anti-hepatic fibrosis single Chinese medicine, traditional Chinese medicine compound active ingredients, pharmacological effects, to clarify the mechanism of anti-hepatic fibrosis of traditional Chinese medicine and the exact action target, is also one of the important tasks of basic research in the future. There is growing evidence that gut dysbiosis aids in the pathogenesis of liver cirrhosis and contributes to the overall systemic inflammatory milieu. Further studies are needed to meaningfully study the functional aspects of these microbial changes that serve as potential mediators of the microbe-host interactions. Similar to the gut microbiome, emerging evidence suggests that the oral microbiome influences changes in the liver; therefore, having a better understanding of the oral-gut-liver represents an exciting new research frontier [35].

\section{References}

[1] Jung YK, Yim HJ. (2017). Reversal of liver cirrhosis: current evidence and expectations [J]. Korean J Intern Med. 2017; 32(2): 213-228.

[2] Chang Hong, Guo Kai, Meng Hongyu, Liu Shumin. (n.d.). Advances in the study of the molecular mechanism of anti-hepatic fibrosis of hepatic stellate cells by the active components of traditional Chinese medicine [J]. Chinese Modern Chinese Medicine 19(01): 148-153.

[3] Xu Xiaoyuan, Ding Huiguo, Li Wengang, Xu Jinghang, Han Ying, Jia Jidong, Wei Lai, Duan Zhongping, Ling Huenqiang, Zhuang Hui. (2019). Guidelines for the diagnosis and treatment of [J]. Cirrhosis Journal of Clinical Hepatobiliary Diseases, 2019, 35(11): 2408-2425.

[4] Wang Guiqiang, Wang Fusheng, Zhuang Hui, Li Taisheng, Zheng Sujun, Zhao Hong, Duan Zhongping, Hou Jinlin, Jia Jidong, Xu Xiaoyuan, Cui Fuqiang, Wei Lai. (2019). Guidelines for the Prevention and Control of Chronic Hepatitis B (2019 Edition) [J]; and Chinese Journal of Medical Frontier (Electronic Edition), 2019, 11(12): 51-77.

[5] Chassaing B, Etienne-Mesmin L, Gewirtz AT. (2014). Microbiota-liver axis in hepatic disease [J]. Hepatology. 2014; 59(1): 328-339.

[6] Lata J, Jurankova J, Kopacova M, et al. (2011). Probiotics in hepatology [J]. World J Gastroenterol. 2011; 17(24): 2890-2896.

[7] Li Yanling, Zhang Huaihong, Zhai Yufeng. (2014). Effect of Probiotics on Hepatocyte Apoptosis in Cirrhotic Rats [J]. Chinese Journal of Gerontology, 2014, 34(03): 708-710.

[8] Chen Yihua. (n.d.). Application value of probiotics in the treatment of liver cirrhosis [J]. Northern Pharmacy 16(09): 113-114.

[9] Yu Shuxia. (2004). Effects of intestinal permeability and probiotics in cirrhotic patients [C]. and Chinese Society of Integrated Chinese and Western Medicine liver disease professional committee. Compilation of papers of the Thirteenth National Conference on Integrated Chinese and Western Medicine liver Disease. Chinese Society of Integrated Chinese and Western Medicine liver disease professional committee: China Society of Integrated Chinese and Western Medicine 2004:83-87.

[10] That Yan, Lou Xianzhi. (2019). Clinical efficacy of microbial agents combined with entecavir in the treatment of hepatitis B cirrhosis [J]. Journal of China Medical University, 2019, 49(2).

[11] Borrelli A, Bonelli P, Tuccillo F M, et al. (2018). Role of gut microbiota and oxidative stress in the progression of non-alcoholic fatty liver disease to hepatocarcinoma: Current and innovative therapeutic approaches [J]. Redox Biology, 2018, 15: 467-479.

[12] Wang Rong, Xie Lingli, Song Mingyu. (2019). Advances in the study of the effects of gut microbiota on chronic liver disease [J\O].]. Chinese Journal of Integrated Chinese and Western Medicine digestion, 2019, 27(11), 879-882.

[13] Usami M, Miyoshi M, Yamashita H. (2015). Gut microbiota and host metabolism in liver cirrhosis. World J Gastroenterol. 2015; 21(41): 11597-11608.

[14] Tripathi A, Debelius J, Brenner DA, et al. (2018). The gut-liver axis and the intersection with the microbiome [published correction appears in Nat Rev Gastroenterol Hepatol. 2018 May 21]. Nat Rev Gastroenterol Hepatol. 2018, 15(7): 397-411.

[15] Zhu Yun, Cheng Jia Dai, Wang Lifu, Yang Huiyin, Wang Zhongxia, Zhou Kun, Xiao Xiaohe, Li Yonggang. (n.d.). An overview of Chinese medicine in the treatment of drug-induced liver injury [J]. Journal of Hepatology with Integrated Chinese and Western Medicine 24(04): 254-256.

[16] Chang Hong, Guo Kai, Meng Hongyu, Liu Shumin. (n.d.). Advances in the study of the molecular mechanism of anti-hepatic 
fibrosis of hepatic stellate cells by the active components of traditional Chinese medicine [J]. Chinese Modern Chinese Medicine 19(01): 148-153.

[17] Wang Dianbing, Li Hengfei, Lu Dingbo. (2018). Clinical Effect of TCM Comprehensive Treatment Scheme on Compensatory Liver Cirrhosis [J]. World’s Latest Medical Information Digest, 2018, 18(86): 120-122.

[18] Peng, Chen Liang, Jiang Xiaoyan, Xing Yufeng, Jiang Mingjie, Tong Guangdong, Zhou Bridge. (n.d.). Experience of Professor Zhou Daqiao in the Treatment of Early Cirrhosis Journal of liver Disease with Integrated Chinese and Western Medicine 28(03): 183-185.

[19] Cai FF, Wu R, Song YN, et al. (2018). Yinchenhao Decoction Alleviates Liver Fibrosis by Regulating Bile Acid Metabolism and TGF- $\beta /$ Smad/ERK Signalling Pathway [J]. Sci Rep. 2018; 8(1): 15367.

[20] Cai FF, Bian YQ, Wu R, et al. (2019). Yinchenhao decoction suppresses rat liver fibrosis involved in an apoptosis regulation mechanism based on network pharmacology and transcriptomic analysis [J]. Biomed Pharmacother. 2019; 114: 108863.

[21] Li Musong, Tian Yanhong, Zhao Yuqian, Yang Huixuan. (n.d.). Advances in Clinical Research of Yinchenhao Decoction [J]; and Chinese folk medicine 27(16): 51-53.

[22] Zhu Yun, Cheng Jia Dai, Wang Lifu, Yang Huiyin, Wang Zhongxia, Zhou Kun, Xiao Xiaohe, Li Yonggang. (n.d.). An overview of Chinese medicine in the treatment of drug-induced liver injury [J]. Journal of Hepatology with Integrated Chinese and Western Medicine 24(04): 254-256.

[23] Hu Yanwu, Liu Kai, Yan Mengtong, Wang Lei, Liu Mingdong, Yu Junlin, Wu Zijing. (n.d.). Effect of stem extract of Schisandra chinensis on liver fibrosis in rats [J]. Its mechanism Chinese Journal of Experimental prescriptions 22(17): 122-125.

[24] Yu Huanghe, Li Xin, Yang Zhen, Lin Yi, Yan Jianye, Wang Yuanqing, Zeng Rong, Wang Wei, Cai Xiong. (n.d.). Advances in Pharmacological Action of Schisandra chinensis [J]. Universal Chinese Medicine 12(07): 1133-1138.

[25] Chen Q, Zhang H, Cao Y, et al. (2017). Schisandrin B attenuates CCl4-induced liver fibrosis in rats by regulation of Nrf2-ARE and TGF- $\beta$ /Smad signaling pathways [J]. Drug Des Devel Ther. 2017; 11: 2179-2191.

[26] Lin Hui, Mei Quanxi, Kong Xianglian, Wang Yunting, Chen Zongliang. (2011). Clinical Application and Mechanism of Hedyotis diffusa in the Treatment of Hepatic Disease [J]. A Asia Pacific Traditional Medicine, 2011, 7(10): 173-175.

[27] Cao Haifang, Zu Hongmei, Peng Junning, Wang Hude, Han Yun, Lu Qinghua. (n.d.). Effect of Salvia miltiorrhiza combined with tenofovirate on liver protection in hepatitis B cirrhosis [J]. Chinese Journal of traditional Chinese Medicine 36(07): 1679-1682.

[28] Lu Zhenzhen, Su Qijian, Li Xuan, Fu Linchun. (2015). Comparison of efficacy and drug resistance analysis of high-efficiency antiretroviral therapy with tenofovir or zidovudine [J]. Journal of Applied Medicine, 2015, 31(09): 1475-1478.

[29] Xia Hua. (2017). Effects of Compound Danshen Tablets Combined with Nucleoside Antiviral Drugs on Fibrosis Indexes in Hepatitis B Cirrhosis Patients [J]. Chinese convalescent medicine, 2017, 26(08): 865-866.

[30] Jiang Hongwei. (2007). 30 Cases of Hepatitis Cirrhosis Treated by Single Flavor [J]. Biejia Clinical Medicine, 2007(06): 93-94.

[31] Sun Zhenliang, Wu Zhe, Guixiangping, Tian Lingyun, Li Xianpeng. (2019). Effect of Compound Biejia Softgan Tablets on Liver Fibrosis Rats and Journal of Yunnan College of traditional Chinese Medicine, 2019, 42(01): 30-58.

[32] Huangfu Yanlin, Wu Huikun. (2019). Advances in the Treatment of Cirrhosis with Chinese Medicine [J]. Journal of Liaoning University of Traditional Chinese Medicine, 2019, 21(07), 221-224.

[33] Lin LJ, Chen GF, Gu HT, et al. (2014). Performance of Fibro Scan in evaluating the curative effects of tradition Chinese medicine on liver fibrosis [J]. Chin J Hepatol, 2014, 22(2): 113-117.

[34] Li Aizhi, Feng Xianni, Zhang Chunju, et al. (2012). Treatment of 25 cases of chronic hepatitis B with adefoviride [J]. Chronic hepatitis B early cirrhosis Traditional Chinese Medicine Research, 2012, 25(3): 28-30.

[35] Gu Shaoyu. (2013). Clinical Observation on 72 Cases of Early Liver Cirrhosis Treated by Self-Drafting Qing Liver Decoction [J].] Inner Mongolia Chinese Medicine, 2013, 32(2): 4.

[36] Acharya C, Sahingur SE, Bajaj JS. (2017). Microbiota, cirrhosis, and the emerging oral-gut-liver axis. JCI Insight. 2017; 2(19): e94416. Published 2017 Oct 5. 